Dear Editor,

\title{
Comments on the second letter of Weiner [11]
}

I do not accept the proof of Weiner's new lemma, because he uses Lemma 2 of his paper [9]. In fact, he says that similar arguments, along with Lemma 2, establish (1.4), (1.5). The point of my criticism [4] on Lemma 2 in common with Tanemura's [7] and Tory and Pickard's [8] was that Weiner's assertion that 'the horizontal placement and parking of cars on the line segment $l$ is independent of all other parked cars and depends only on the $x$-coordinate' is false. As stated by Tory and Pickard [8], it is true that the coordinates of attempted placements are chosen independently, but the success or failure of the attempt depends on the positions of cars already in place. In Weiner's proof of Lemma 2 and his reply [10] to our criticisms, he only claimed independence but did not prove it.

Furthermore, I cannot understand why Lemma 2 leads to (1.4) and (1.5). It seems to me that Lemma 2 is not sufficient to derive (1.4) and (1.5). He should have described the process of reasoning in more detail and more definitely.

Even if the new lemma is correct, it is not assured that his new theorem holds true. In all patterns of packing in accord with his scheme in the proof of the theorem, there exist a number of cars whose sides have the same abscissa (e.g., $x=\xi$ ) or the same ordinate (e.g., $y=\eta$ ). In the case of random packing in Rényi's sense, however, the event that two or more cars have sides with the same $x$ - (or $y$-) coordinate does not occur with probability one. It is also noted that in Weiner's case there exist no cars with sides whose $x$-coordinate satisfies $\xi<x<\xi+1$, while in Rényi's case the probability of cars being parked in the same region is non-zero. Therefore, the process considered by Weiner is essentially different from the Rényi model.

Finally, the term $m(a)+m(b)-1$ on the right-hand side of (1.6), (1.7) seems erroneous. Weiner miscounts the possibility of overlapping of cars parked in the horizontal and vertical strips. In the pattern shown in Figure 1, for example, the

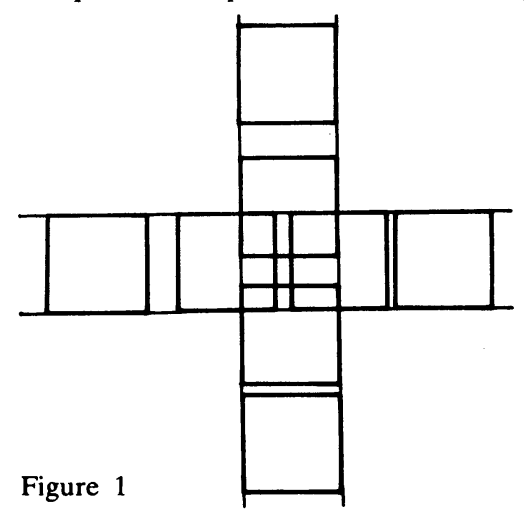

Received 23 November 1979; revision received 4 January 1980. 


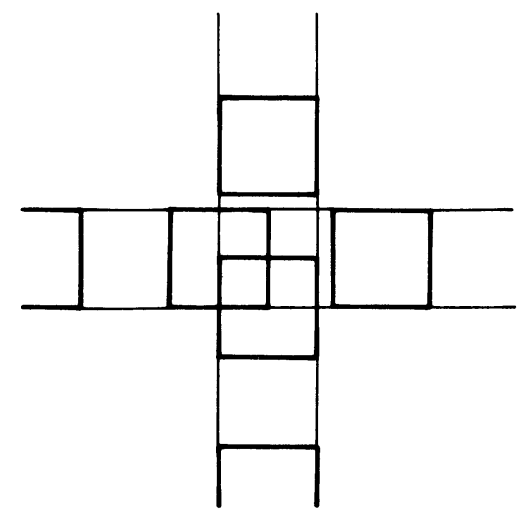

a

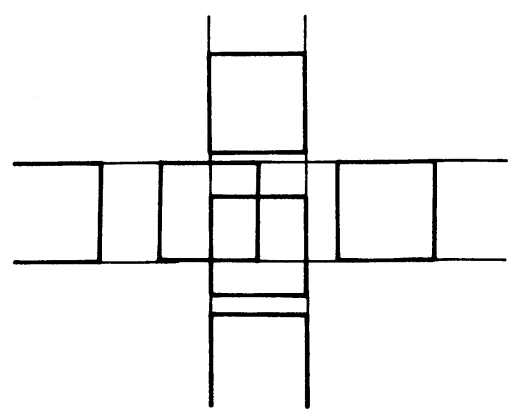

a

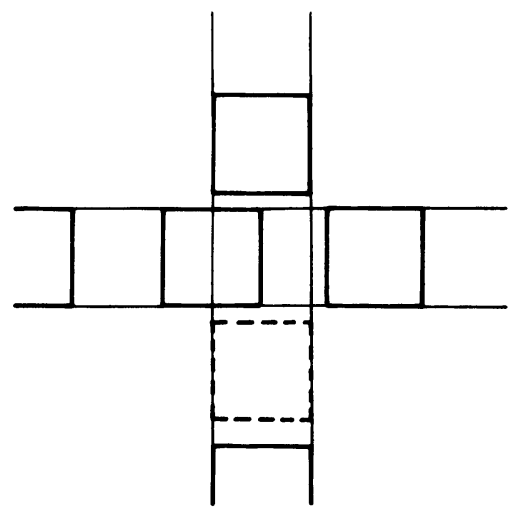

b

Figure 2

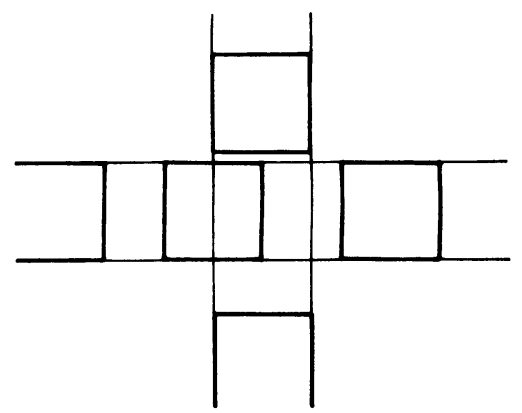

b

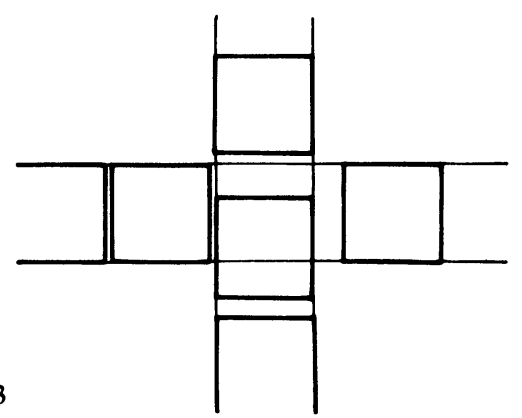

Figure 3

number of cars that should be omitted is not 1 but 2 . For the case illustrated in Figure 2, on the other hand, we must delete a car and then add another car. Besides, the number of cars to be omitted cannot be uniquely determined in some cases. Is the number of cars to be omitted in Figure 31 or 0? Let us tentatively take the expectations for possible numbers and assume that gaps between cars are independent and uniformly distributed; then the mean number of cars after discarding is found to be greater than $m(a)+m(b)-1$.

Tokyo Institute of Technology

Yours sincerely

MoTOo HoRI 
Note added in proof. A two-dimensional packing model for which the Palásti conjecture is true has recently been proposed by I. Palásti herself. (On a two-dimensional random space filling problem. Studia Sci. Math. Hungar. 11 (1976), 247-252.) In her packing scheme, the sides of the car placed at first are prolonged horizontally and vertically, and the bands with unit width thus obtained are filled in accordance with the one-dimensional packing law. The processes of placing subsequent cars in the domains separated by those bands are mutually independent. Therefore, her procedure is essentially of onedimensional character and quite different from the usual two-dimensional packing in Rényi's sense.

Dear Editor,

\section{Reply to remarks of Professor M. Hori}

The numbers at the beginning of a paragraph refer to the numbers in Professor Hori's letter (pp. 888-890 of this issue).

1. The 'top row' as given in Lemma 2 [9] must have horizontal spacing of parked cars as in the one-dimensional case since the $x, y$ coordinates of attempted placements are independent, and the top row must be filled so that no further car can be parked. This appears equivalent to the one-dimensional model.

2. By the mapping, the top row of width $b$ of the $a \times b$ rectangle has, considering only the horizontal coordinates, by Lemma 2 of [9], cars distributed as in a one-dimensional Rényi model, with expectation $m(b)$. By the mapping in [10], condensing the cars in the top row to fit into a horizontal strip $1 \times b$ only increases their density. Hence (1.4), (1.5) of [10] follow.

3. The method used to consider the Rényi and Solomon parking methods in the plane consists in comparing each model with a new parking model in each case for which integral inequalities can be written and solved asymptotically, and which are used to provide upper and lower bounds for the mean total numbers of cars parked in the Rényi and Solomon models, respectively. This replacement model consists in placing two perpendicular strips intersecting at the first car parked, each strip wide enough to accommodate one car, and reaching to the horizontal or vertical sides of the parking rectangle (see Figure 1 of [9]). For an

Received 22 January 1980.

Partial support ONR Contract N00014-76-C-0475 and U.S. Army Research Grant DAAG29-77-G-0031 at Stanford University. 